\title{
Klinische Prüfung eines neuen photometrischen Tests zur Bestimmung der Faktor XIII-Aktivität im Plasma
}

\author{
Clinical Evaluation of a New Photometric Test for Factor XIII Activity in Plasma \\ Eva Solleder, Dagmar Domuth, C. Pfeiffer, M. Bomhard, J. Mayer, Th. Eller, P. Brauer, F. Keller, J. Grün*, K. Fickenscher*, \\ Carola Wagner" \\ Zentrallabor der Medizinischen Universitätsklinik Würzburg \\ - Behringwerke AG, Marburg
}

\begin{abstract}
Zusammenfassung:
Ein neu entwickelter photometrischer Test (Berichrom F XIII) zur Bestimmung der Faktor-XIII-Aktivität im Plasma wurde sowohl am Cobas Fara als auch in der manuellen Version am Spektralphotometer geprüft. Bei diesem Test wird die durch NADH-Verbrauch verursachte Absorptionsabnahme bei $340 \mathrm{~nm}$ gemessen. Das $\mathrm{NADH}$ wird während der durch Glutamatdehydrogenase katalysierten Inkorporation des mittels einer $F$ XIIlaReaktion freigesetzten $\mathrm{NH}_{3}$ in $\alpha$-Ketoglutarat verbraucht. Die Absorptionsabnahme ist porportional zur $F$ XIIIAktivität in der Probe.

Der Test zeichnet sich durch eine hohe Präzision, eine gute Korrelation mit dem Clot-Lyse-Test, durch seine Unempfindlichkeit gegen Störeinflüsse, seine Schnelligkeit und einfache Durchführbarkeit aus. Er wird zu einer enormen Erleichterung der F XIII-Bestimmungen in den klinischen Labors führen.
\end{abstract}

\section{Schlüsselwörter:}

Faktor XIII-Transamidase - Clot-Lyse-Test - NADH-abhängige Bestimmung - Zentrifugalanalyzer

\begin{abstract}
Summary:
A new developed photometric assay (Berichrom F XIII) for the detection of factor XIII activity in plasma was tested. In this assay the decrease in absorbance caused by $\mathrm{NADH}$ consumption due to incorporation of $\mathrm{NH}_{3}$ is detected at $340 \mathrm{~nm}$. This $\mathrm{NH}_{3}$ is released in a $\mathrm{F}$ XIlla catalyzed reaction into $\alpha$-ketoglutarate with the aid of glutamate dehydrogenase. The maximal rate of this decrease in absorbance at $340 \mathrm{~nm}$ is proportional to the activity of F XIIla in the sample.
\end{abstract}

This new assay can easily be performed on clinical analyzers or usual photometers. The test reveals a high precision, a good correlation to the clot lysis test and is insensitive to interferences. Due to its rapid and easy performance this assay will contribute to simplify determinations of F XIII in clinical laboratories.

Keywords:

Factor XIII-transamidase - clot lysis test - NADH-dependent detection - centrifugal analyzer

\section{Einleitung}

Der Faktor XIII gewinnt neben seiner wichtigen Rolle in der Hämostase (Stabilisierung des Fibringerinnsels durch Quervernetzung des löslichen Fibrins zu nichtlöslichen Polymeren, Verminderung der fibrinolytischen Aktivität durch Einlagerung von $\alpha_{2}$-Antiplasmin in das gebildete Fibrin) zunehmend an Bedeutung bei der Behandlung von Wundheilungsstörungen $(1,2,3,4,5)$, Bindegewebserkrankungen wie 2 . B. Sklerodermien $(6,7,8)$, Vaskulitiden wie Schönlein-Henoch-Purpura $(9,10,11,12)$, entzündlichen Darmerkrankungen, wie Morbus Crohn und Colitis ulcerosa $(13,14,15,16)$, Leukämien $(17,18)$, Subarachnoidalblutungen $(19,20)$ sowie bei der Förderung der Knochenheilung $(2,21,22,23,24,25)$.

Dies führt zu einer ständig steigenden Zahl der Anforderungen von Faktor XIII-Bestimmungen. Da die bisher zur Verfügung stehenden Tests zur Bestimmung der Faktor XIII-Aktivität jedoch oft ungenau, zeitintensiv, sehr umständlich in der Durchführung und nicht automatisierbar sind, wurden in letzter Zeit verstärkt Anstrengungen unternommen, neue Testverfahren wie Mikrotiter-Assays
$(26,27,28)$ oder photometrische Tests $(29,30)$ zu entwikkeln.

Ein neuer photometrischer Test (Berichrom F XIII), bei welchem durch Zusątz eines speziell entwickelten Clotinhibitors die Defibrinierung entfällt, wurde von uns geprüft und mit dem im Routinelabor üblicherweise durchgeführten Clot-Lyse-Test verglichen. Zusätzlich wurde im Rahmen des Methodenvergleichs bei 47 Plasmen die Faktor XIII-Aktivität außer mit Berichrom $F$ XIII mit einem zweiten, photometrischen Test (Clinisotest Factor XIII) bestimmt.

\section{Material und Methoden}

\section{2:1 Ṕlasmaproben}

Alle untersuchten Blutproben stammten von Patienten der Medizinischen Universitätsklinik Würzburg. Das Blut wurde in Gerinnungsmonovetten (Sarstedt, Nümbrecht) gewonnen und mit 3,13\%igem Natriumcitrat pH 7,3 antikoaguliert (Mischungsverhältnis Antikoagulans: Blut = $1:$ 10). Nach der Zentrifugation $10 \mathrm{~min}$ bei $2680 \mathrm{~g}$ wurder 


\section{Diagnostitk in Deutschland hat einen guten Namen: Behring}

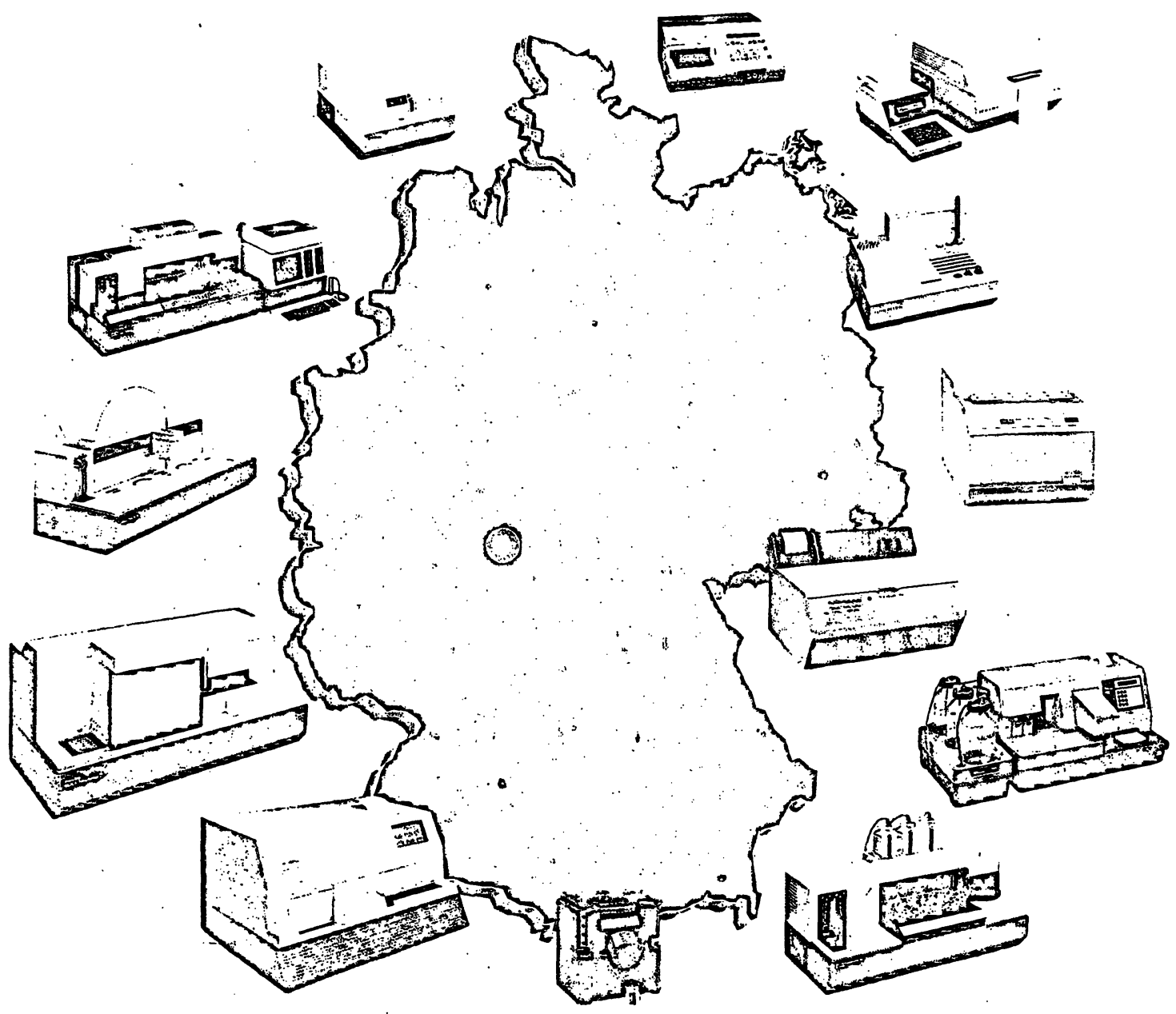

Behring bietet Ihnen ein umfassendes Diagnostika-Programm. Von qualitativen ScreeningTesten für die ärztliche Praxis, die leicht zu handhaben und schnell abzulesen sind, bis zu hochspezifischen automatisierten Systemen für die quantitative Laboratoriumsdiagnostik.

Behring arbeitet intensiv an neuen Entwicklungen, die Ihre Probleme immer intelligenter und exakter lösen.

Sprechen Sie mit uns über 


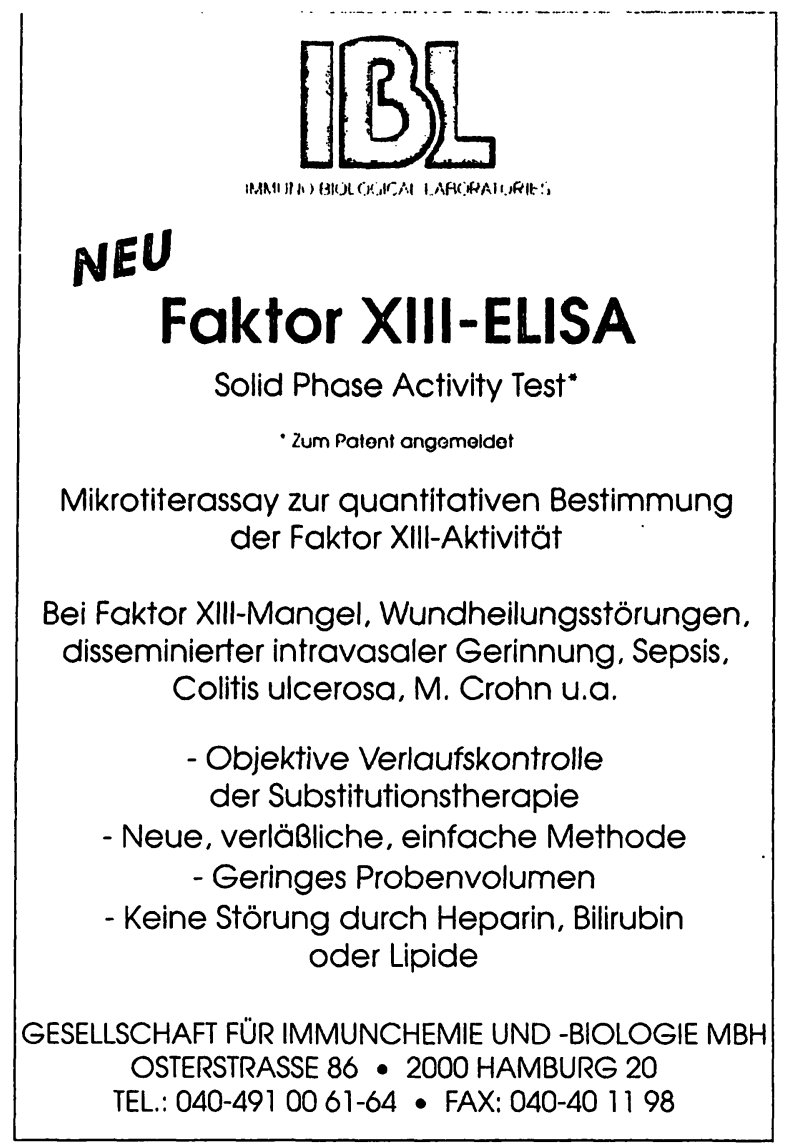

\section{Standardisierte Reagenzien für mikrobiologische Laboratoriums-Arbeiten}

dehydrierle sowie fertigmedien, Seivebezur.t. materistien. Seren und Reogenzien.

Pesistenz-Blattchen, Carbohydrate, Farb:triffe, Anticjeries is a iere.

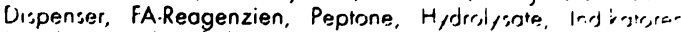
Estratte, Biochemikalien, Aminosaurer, Er.z/me. Jerr,irg ia:ce sowie klinische Reogenzien und Anreirheriung; merd er

(1) I CO $\begin{aligned} & \text { DlFCO-Produkte werden routinemäßig in } \\ & \text { der ganzen Welt verwendet, wo mikro- } \\ & \text { biologische Arbeiten stalfinden. }\end{aligned}$ Sie wurden speziell geschaffen für die blin erom late; torien in Kronkenhäusern. H/giene.Institute. Gevetsezur.laboratorien, Gesundheitsämler - für Wasser ijnd Abum: serkontrollen, Milch- und Lebensmitteluntersuchungen, Qualitäts überwachung in der chem.pharm. Industrie. Erz:eriungs- i-s Ausbildungsinstitute.

\section{ISMUNIT}

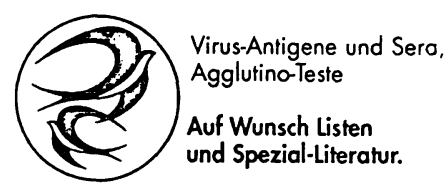

(2)ा? NTER⿴囗十)

Heinrichstraße $5 \cdot 2000$ Hamburg 50 Tel. $040 / 432827 \cdot$ Fax 040/432830

Spezial-Importeur für Labor-Präparate

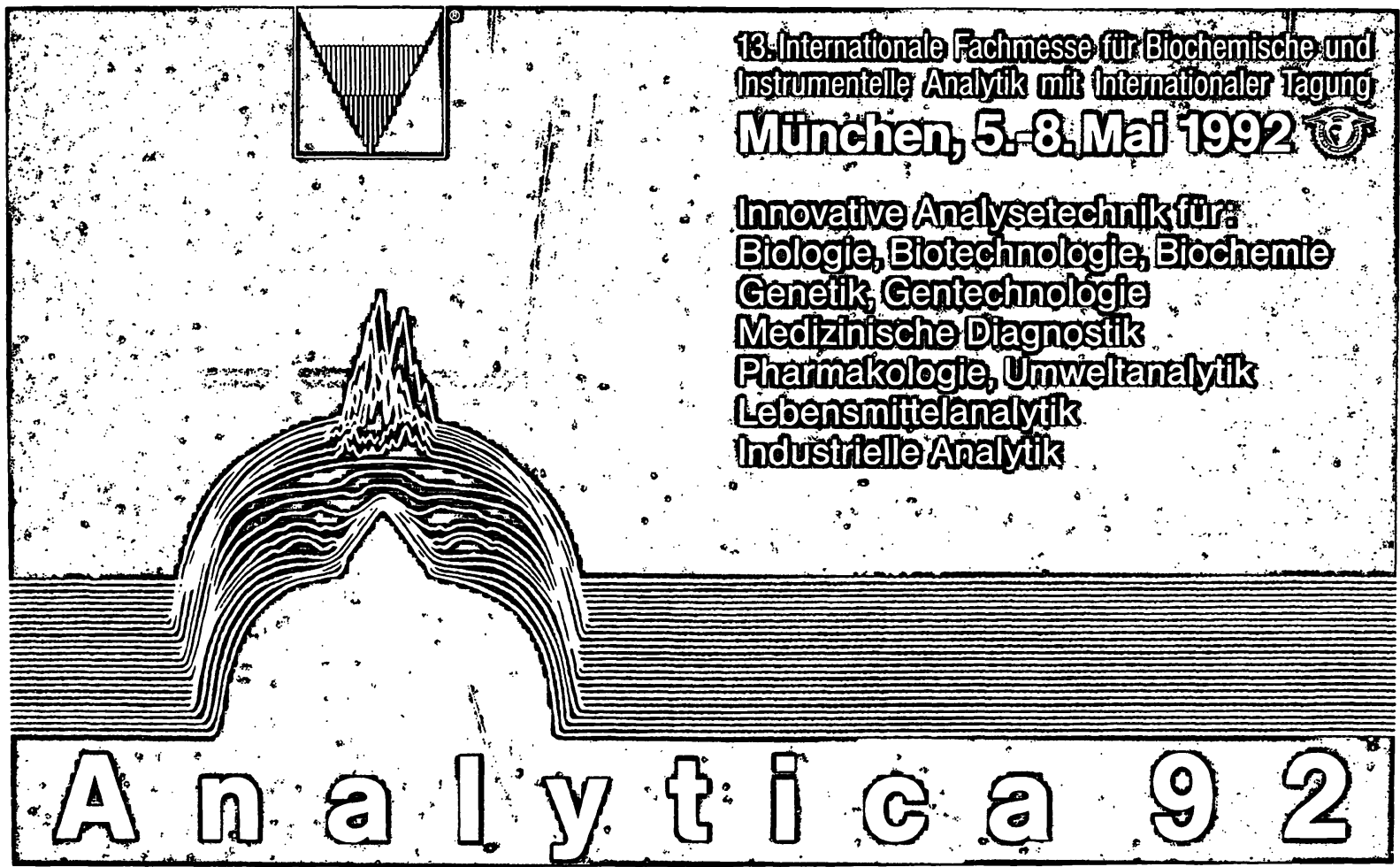


für die Faktor-XIII-Bestimmung Aliquots der gewonnenen Plasmen bei $-18^{\circ} \mathrm{C}$ tiefgefroren. Nur für die Bestimmung des Referenzbereiches wurde der Faktor XIII aus frisch gewonnenen Plasmen bestimmt.

\subsection{Analysengeräte}

Die photometrische F XIII-Bestimmung wurde sowohl am Zentrifugalañalyzer Cobas Fara (Hoffmann La Roche, Basel) als auch am Spektralphotometer LS 500 (Dr. Bruno Lange $\mathrm{GmbH}$, Berlin) durchgeführt.

\subsection{Faktor XIII-Testmethoden}

\subsubsection{Allgemeines Testprinzip der photometrischen F XIII- Bestimmung}

Bei den Methoden handelt es sich - von der Fibrinogenausfällung mit Bentonit beim Clinisotest abgesehen um eine Einstufenmethode, wobei dem unverdünnten Plasma ein Reagenz zugegeben wird, welches alle für die Reaktion notwendigen Komponenten enthält.

Zunächst wird in Gegenwart von $\mathrm{Ca}^{2+}$-lonen der in der Probe vorhandene Faktor XIII durch Thrombin aktiviert. Nach seiner Aktivierung, welche sich in der Reaktionskinetik in einer lag-Phase von ca. 5 min äußert, führt der gebildete Faktor XIIla in einer Transamidasereaktion, bei der die beiden im Reagenz enthaltenen Substate miteinander verknüpft werden, zur Freisetzung von Ammonium-lonen. Nach Zugabe des Reagenz wird die Extinktionszunahme bei $340 \mathrm{~nm}$ verfolgt. Die während dieser Transamidierung gebildeten Ammonium-lonen werden mittels einer Glutamatdehydrogenase - katalysierten, $\mathrm{NAD}(\mathrm{P}) \mathrm{H}$-abhängigen Reaktion gemessen. Im linearen Bereich der Kinetik ist die Extinktionsabnahme direkt proportional zur F XIII-Aktivität (27). In diesem Bereich wird deshalb über einen Zeitraum von 5 min die Extinktionsabnahme pro Minute gemessen und daraus die F XIII-AKtivität im Plasma bestimmt. Bei beiden photometrischen Tests wurde Standard-Humanplasma der Behringwerke AG (Marburg) als Standard eingesetzt.

\subsubsection{Berichrom F XIII-Test}

(Behringwerke AG, Marburg/BRD)

Für den manuellen Test am Spektralphotometer LS 500 wurden gleiche Volumina des Aktivatorreagenz (Rinderthrombin, Gly-Pro-Arg-Pro-Ala-amid, $\mathrm{CaCl}_{2}$, Polybren, BICINE-Puffer $\mathrm{pH}$ 8,3, Albümin, Natriumazid, NADH) und das Nachweisreagenz (Glutamat-Dehydrogenase, Glycinethylester, L-Leucyl-L-Glycyl-L-Prolyl-L-Glycyl-L-Glutaminyl-L-Seryl-L-Lysyl-L-Valyl-L-Isoleucyl-L-Glycyl-amid), ADP, $\alpha$-Ketoglutarat, Hepes-Puffer pH 6,5, Albumin, Natriumazid) zu einem Monoreagenz vermischt und bei $37^{\circ} \mathrm{C}$ inkubiert.

Testansatz in einer auf $37^{\circ} \mathrm{C}$ temperierten Kunststoffküvette:

Probe:

Monoreagenz:

$100 \mu 1$ $1000 \mu l$

Nach dem Mischen und Starten der Messung wurde nach $5 \mathrm{~min}$ und $10 \mathrm{~min}$ die Extinktion abgelesen und $\Delta E /$ $\min$ berechnet.

Am Cobas Fara II wurden Aktivator- und Nachweisreagenz getrennt eingesetzt, wodurch sich die Stabilität des Reagenzes deutlich verlängert.

Testansatz am Cobas Fara II:

Probe:

Aktivatorreagenz:

Nachweisreagenz:
Durch einen "Temp. delay" von $120 \mathrm{sec}$ wurde gewährleistet, daß die Testtemperatur von $37^{\circ} \mathrm{C}$ vor Beginn der Analyse erreicht wird. 5 min nach Beginn der Messung bei $340 \mathrm{~nm}$ wurde in Zeitintervallen von $30 \mathrm{sec} 10 \times$ die Extinktion abgelesen (d. h. die gesamte Meßdauer beträgt $10 \mathrm{~min}$ ).

\subsubsection{Clinisotest F XIII}

(Isotopenlabor Budapest/Ungarn)

Dieser Test entspricht - geringfügig modifiziert - der kinetischen UV-Methode von Muszbek et al. (29). Der eigentlichen Bestimmung geht eine Fibrinogenfällung voraus. Dafür wurden $1,5 \mathrm{ml}$ Citratplasma in Reaktionsgefäße pipettiert, welche $60 \mathrm{mg}$ Bentonit enthalten. Nach einer 10minütigen Inkubation wurde das Plasma erneut zentrifugiert und der fibrinogenfreie Überstand für die F XIII-Bestimmung verwendet.

Da bei dieser Methode von jeder Probe ein Probenleerwert mitgeführt werden muß, wurde für die Leerwertbestimmung das im Reagenz enthaltene $\mathrm{CaCl}_{2}$ durch EDTA ersetzt, um die Aktivierung des im Plasma vorhandenen F XIII zu unterbinden.

Um das gebrauchsfertige Reagenz zu erhalten, müssen zu dem NADPH-Lyophilisat $1 \mathrm{ml}$ des Substratpuffers $(\alpha$ Oxoglutarat, ADP, Dithiothreitol, Et- $\mathrm{NH}_{2}$ (ethylamin) $\mathrm{HCl}$, AD- $\beta$-casein, Hepes-Puffer pH 7,5), $200 \mu$ l Thrombin, 50 $\mu$ l Glutamatdehydrogenase und $100 \mu \mathrm{l} \mathrm{CaCl}_{2}$ (bzw. für den Probenleerwert EDTA) pipettiert werden. Beim Testansatz am Cobas Fara II wurden zu $90 \mu \mathrm{l}$ Probenvolumen im 1. Lauf $135 \mu \mathrm{l}$ Reagenz pipettiert und nach 5 minütiger Inkubation in Zeitintervallen von $60 \sec 4 \times$ die Extinktion gemessen. Für die Leerwertbestimmung wurde im Reagenz das $\mathrm{CaCl}_{2}$ durch EDTA ersetzt und ein 2. Lauf durchgeführt.

\subsubsection{Clot-Lyse-Test}

Als Vergleichsmethode diente der Clot-Lyse-Test mit dem Gerinnungsfaktor XIII-Schnellreagenz der Behringwerke AG, Marburg (31). Es wurden 4 Verdünnungsstufen angesetzt $(1: 5,1: 10,1: 15,1: 20)$, die einem $F$ XIII-Gehalt von $25 \%, 50 \%, 75 \%$ und $100 \%$ d. N. entsprechen und der Test gemäß der Testanleitung durchgeführt.

\section{Ergebnisse}

\subsection{Präzision}

Intra- und Interassay wurden mit einer standardisierten Kontrolle im Normalbereich, einer standardisierten Kontrolle im pathologischen Bereich, sowie einem selbsterstellten Normalpool und einem durch Verdünnung mit $0,9 \% \mathrm{NaCl}$ gewonnenen Plasmapool im pathologischen Bereich durchgeführt.

Bei der Präzision in der Serie $(n=20)$ lagen die Variationskoeffizienten der Normalplasmen am Cobas Fara zwischen 1,8 und 2,0\%, bei der manuellen Methode um $3,1 \%$. Für die Plasmen im pathologischen Bereich ergaben sich am Cobas Fara Variationskoeffizienten zwischen 1,9 und $2,4 \%$, am Spektralphotometer zwischen 3,0 und $6,0 \%$ (Tab. 1).

Von Tag zu Tag $(n=10)$ betrugen die Schwankungen am Cobas Fara ca. $2,8 \%$ bzw. 2,4-4,8\%, bei der manuellen Methode ca. 3,5\% bzw. 3,7-5,3\% (Tab. 2).

\subsection{Referenzbereich}

Für die Bestimmung des Referenzbereichs.wurde der Faktor XIII-Gehalt in 100 Plasmen mit Quick-, PTT- und Fibrinogenwerten im Normalbereich gemessen. Der Mittel- 
Tab. 1: Präzision in der Serie $(n=20)$ am Cobas Fara $(C)$ und am Spektralphotometer (M). 1 = CTS-Kontrollplasma N, $2=$ CTS. Kontrollplasma P 1, 3 = Plasmapool im Normalbereich, 4 = Plasmapool im pathologischen Beraich.

\begin{tabular}{lrrrrrrrr}
\hline & \multicolumn{1}{c}{$\mathbf{C}^{1}$} & \multicolumn{1}{c}{$\mathbf{M}^{2}$} & \multicolumn{1}{c}{$\mathbf{C}^{\mathbf{M}}$} & \multicolumn{1}{c}{$\mathbf{C}^{3}$} & $\mathbf{M}$ & $\mathbf{C}^{4}$ & $\mathbf{M}$ \\
\hline Mittelwert (\%) & 92,5 & 93,9 & 52,8 & 54,6 & 107,0 & 116,8 & 58,7 & 59,1 \\
Std.abweichung & 1,8 & 2,9 & 1,0 & 1,7 & 1,9 & 3,7 & 1,4 & 3,6 \\
Var.koeff. (\%) & 2,0 & 3,1 & 1,9 & 3,0 & 1,8 & 3,1 & 2,4 & 6,0 \\
\hline
\end{tabular}

Tab. 2: Variation von Tag zu Tag ( $n=10$; Doppelbestimmung) am Cobas Fara (C) und am Spektralphotometer (M). $1=$ CTSKontrollplasma N, 2 = CTS-Kontrollplasma P1, 3 = Plasmapool im Normalbereich, 4 = Plasmapool im patholog. Bereich.

\begin{tabular}{lrrrrrrrr}
\hline & \multicolumn{1}{c}{$\mathbf{C}^{\mathbf{1}}$} & $\mathbf{M}$ & \multicolumn{1}{c}{$\mathbf{C}^{2}$} & $\mathbf{M}$ & $\mathbf{C}^{2}$ & $\mathbf{M}$ & \multicolumn{1}{c}{$\mathbf{C}^{\mathbf{4}}$} & $\mathbf{M}$ \\
\hline Mittelwert (\%) & 94,9 & 94,8 & 54,9 & 54,5 & 111,2 & 114,8 & 57,8 & 59,1 \\
Std.abweichung & 2,8 & 3,0 & 1,3 & 2,0 & 3,1 & 4,4 & 2,8 & 3,1 \\
Var.koeff. (\%) & 2,9 & 3,2 & 2,4 & 3,7 & 2,8 & 3,8 & 4,8 & 5,3 \\
\hline
\end{tabular}

wert dieser Proben lag bei $99,8 \%$, der Referenzbereich wurde zwischen $60 \%$ und $133 \%$ (2,5 bis 97,5 Perzentile) festgelegt.

\subsection{Linearität}

Zur Überprüfung der Linearität wurden Proben mit hohem $F$ XIII-Gehalt schrittweise mit $0,9 \% \mathrm{NaCl}$ verdünnt $(10+0 \ldots 1+9)$, um so den gesamten Meßbereich abzudecken. Wie in Abbildung 1 dargestellt, zeigte sich über den gesamten Meßbereich eine gute Korrelation $(r=1,0)$ zwischen dem theoretischen Wert und dem tatsächlich gemessenen Ergebnis.

\subsection{Methodenvergleich}

Der Vergleich der Berichrom F XIII-Bestimmung mit dem Clot-Lyse-Test wurde mit 81 Plasmen durchgeführt, die sich aus folgenden Patientenkollektiven zusammensetzten:

15 Patienten postoperativ

24 Patienten mit Frakturen

34 Patienten mit malignen Erkrankungen

6 Patienten mit Leberdysfunktionen

2 Patienten mit DIC/hämorrhag. Schock

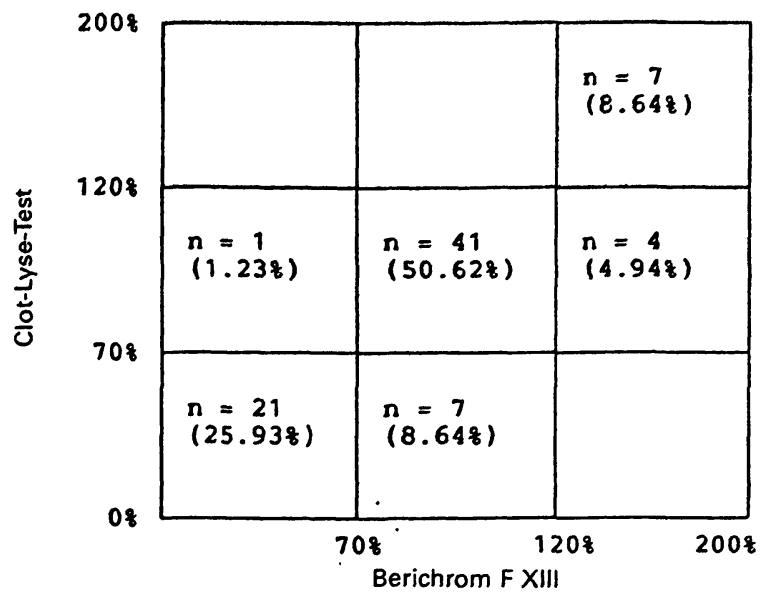

Abb. 2: Auswertung des Methodenvergleichs $(n=81)$ der Faktor XIII-Bestimmung zwischen Berichrom F XIII-Test und Clot-LyseTest mit einer Neunfeldertafel. Die Grenzen $70 \%$ und $120 \%$ d. N. entsprechen dem Referenzbereich.

Von den 81 Wertepaaren waren bei der Auswertung mit der Neunfeldertafel (Abb. 2) 69 Paare konkordant und 12 diskordant. Wertet man aufgrund der semiquantitativen Bestimmung die Korrelation für den Clot-Lyse-Test mit einem Graubereich bis $50 \%$ aus, so fallen in diesen Graubereich weitere 7 Wertepaare. Somit reduziert sich die Zahl der tatsächlich diskordanten Ergebnisse auf 5 $(6,2 \%), 7$ Wertepaare $(8,6 \%)$ können als grenzwertig diskordant bezeichnet werden, die verbleibenden 69 Paare $(85,2 \%)$ sind konkordant.

Zur Klärung methodenspezifischer Unterschiede wurde die F XIII-Aktivität von 56 Proben am Cobas Fara und am Spektralphotometer bestimmt. Bei der Auswertung nach Passing \& Bablok zeigte sich eine gute Korrelation $\langle r=$ 0,988), wobei die mit der manuellen Methode bestimmten Werte etwas höher lagen als die am Cobas Fara erzielten Werte (Abb. 3). Der Methodenvergleich des Berichrom $F$ XIII-Test mit dem Clinisotest ergab eine starke Streuung der Werte (Abb. 4) und mit $r=0,650$ eine schlechte Korrelation.

Von 49 Plasmen wurden jeweils $1,5 \mathrm{ml}$ entnommen, um das Fibrinogen mit Bentonit auszufällen und sowohl in
Abb. 1: Graphische Auswertung (Pasșing \& Bablok) der Linearität zwischen gemessenem (Cobas Fara) und theoretischem F XIII-Wert der Probenverdünnungen.

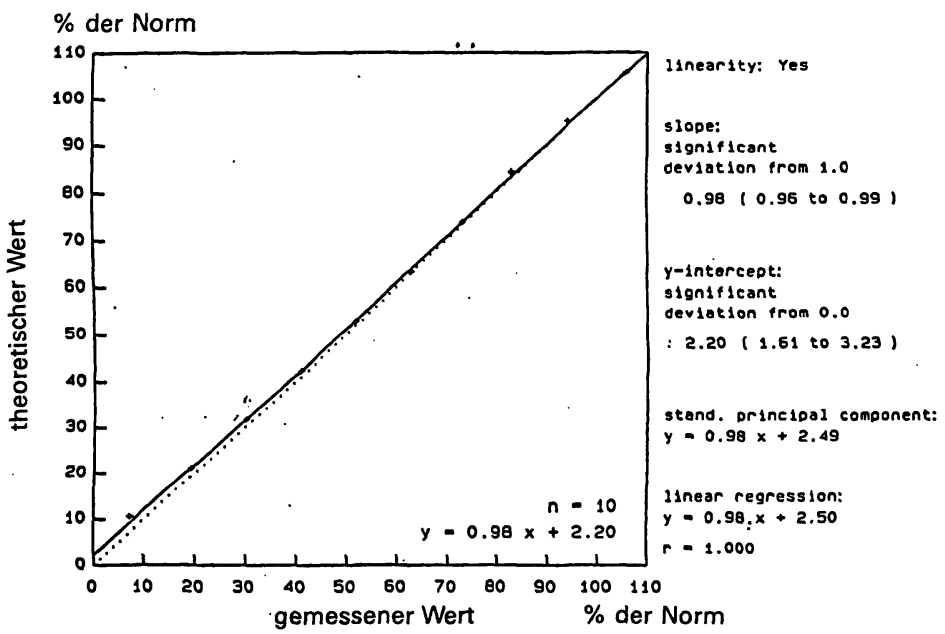


dieser fibrinogenfreien Probe als auch im unbehandelten Plasma die Faktor XIII-Aktivität mit Berichrom F XIII bestimmt. Es zeigte sich, daß in der fibrinogenfreien Probe nur ca. $1 / 4$ der im unbehandelten Plasma nachgewiesenen F XIII-Aktivität gemessen werden konnte (Abb. 5). Dies läßt vermuten, daß neben dem Fibrinogen auch ein Teil des Faktor XIII mit Bentonit ausgefällt wird.

\subsection{Störfaktoren}

Im Plasma erfolgt die Aktivierung von Faktor XIII durch Thrombin in Gegenwart von $\mathrm{Ca}^{2+}(32,33,34)$. Da im Plasma vorhandenes Heparin zusammen mit AT III zu einer Inaktivierung von Thrombin führt, wird somit in heparinhaltigen Plasmen der Faktor XIII nur unvollständig

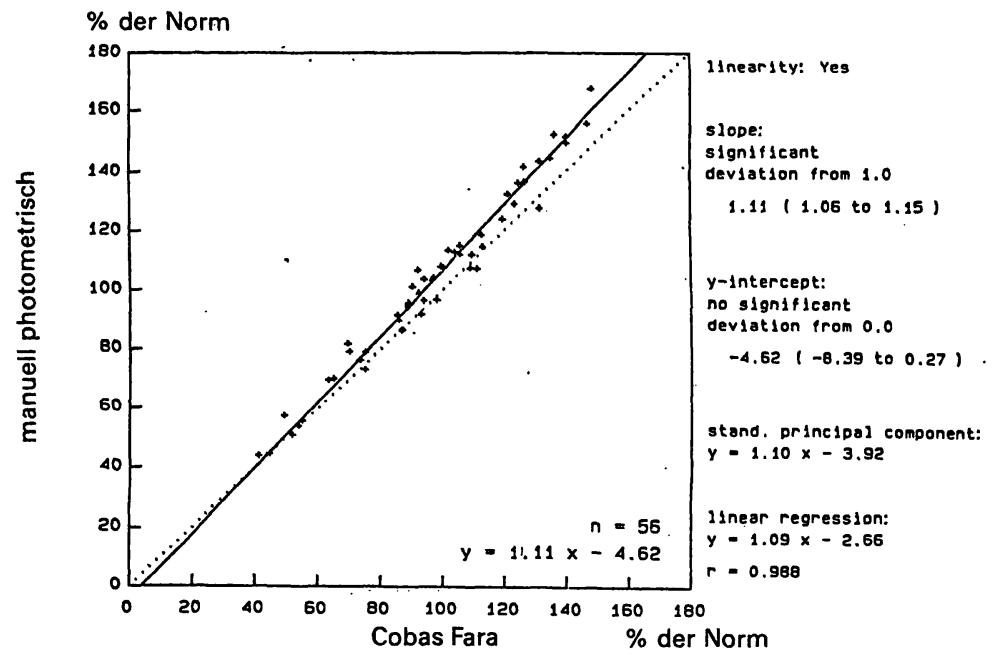

Abb. 3: Graphische Auswertung (Passing \& Bablok) des Methodenvergleichs ( $n=$ 56) des Berichrom F XIII-Tests zwischen der automatisierten (Cobas Fara) und der manuellen (Spektralphotometer) Version.

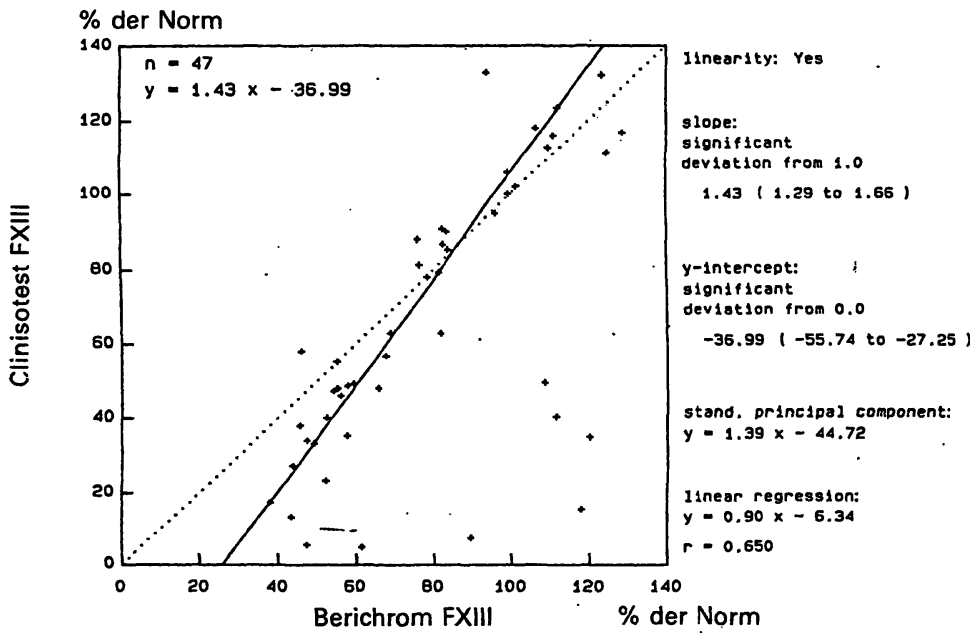

Abb. 4: Graphische Auswertung (Passing \& Bablok) des Methodenvergleichs der Faktor XIII-Bestimmung am Cobas Fara zwischen der Berichrom F XIII-Methode und dem Clinisotest F XIII.

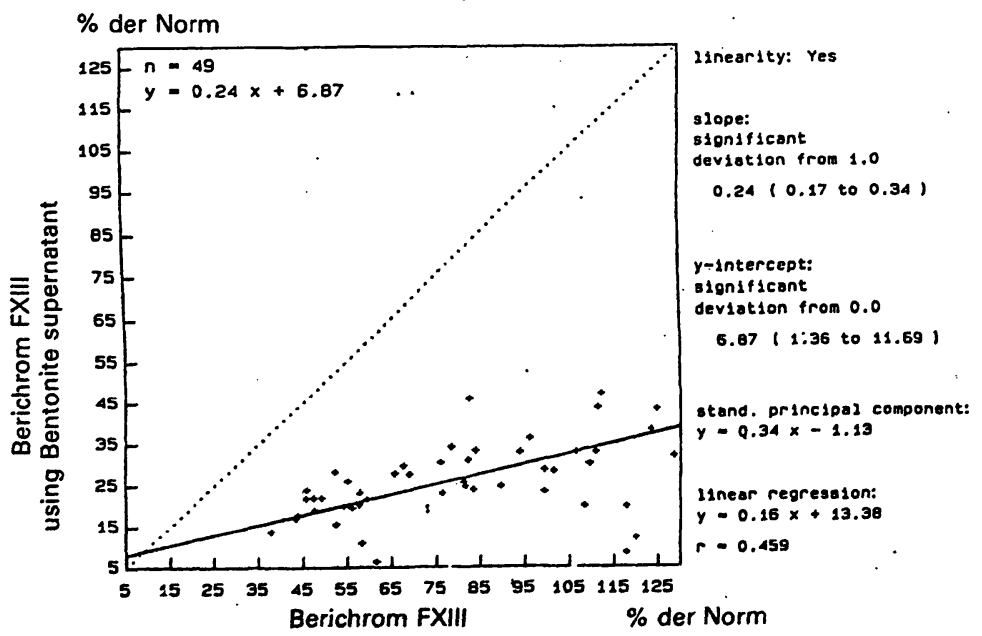

Abb. 5: Graphische Auswertung (Passing \& Bablok) des Methodenvergleichs der Berichrom F XIII-Bestimmung zwischen unbehandelten und mit Bentonit defibrinierten Plasmen. 


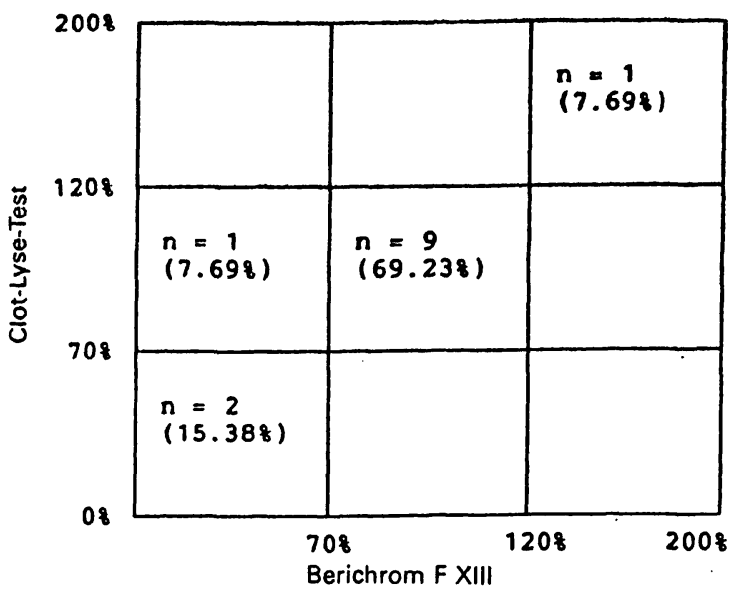

Abb. 6: Auswertung der mit Berichrom F XIII und dem Clot-LyseTest bestimmten F XIII-Werte von 13 Patienten unter Heparintherapie 115000 IE/d) mit einer Neunfeldertafel. Die Grenzen $70 \%$ und $120 \%$ d. N. entsprechen dem Referenzbereich.

in F XIIla umgewandelt. Deshalb wurde dem Aktivatorreagenz des Berichromtest Polybren (ein Polykation) zugesetzt, um die Heparinaktivität bis $1,5 \mathrm{U} / \mathrm{ml}$ zu neutralisieren (30). In den Plasmen von 13 Patienten unter Heparintherapie (15000 IE/d) wurde der Faktor XIII sowohl mit Berichrom F XIII als auch mit dem Clot-Lyse-Test bestimmt. Die Auswertung mit der Neunfeldertafel ergab, daß 12 der 13 Wertepaare konkordant waren, ein Wertepaar war diskordant (Abb. 6). Erweitert man die Neunfeldertafel um einen Graubereich bis $50 \%$, so fällt das diskordante Wertepaar in diesen Bereich und ist somit grenzwertig. Da beim Berichromtest die Faktor XIII-Aktivität über die Messung des Verbrauchs des in der Transglutaminasereaktion gebildeten Ammoniaks erfolgt, kommen erhöhte Ammoniakkonzentrationen im Plasma als weitere Störfaktoren in Frage. Deshalb wurde in 11 Plasmen mit erhöhten Ammoniakwerten (93-226 $\mu \mathrm{g} / \mathrm{dl}$ ) die Faktor XIIIBestimmung mit Berichrom F XIII und dem Clot-Lyse-Test durchgeführt. 8 der 11 Wertepaare sind nach der Auswertung mit der Neunfeldertafel konkordant, 3 sind diskordant, jedoch bei der Auswertung in einer erweiterten Neunfeldertafel mit einem Graubereich bis $50 \%$ als grenzwertig zu bezeichnen (Abb. 7).

\section{Diskussion}

Durch die ständig steigende Zahl der F XIII-Bestimmungen wird die Forderung nach einfacheren Routinetests immer dringlicher. Um zu einer einfachen und schnellen Bestimmungsmethode zu gelangen, wie dies z. B. im Berichrom $F$ XIII-Test verwirklicht ist, mußten jedoch eine ganze Reihe von Problemen gelöst werden (30). So wird z. B. der im Plasma in einer inaktiven Vorstufe vorliegende $\mathrm{F}$ XIII durch Thrombin in Gegenwart von $\mathrm{Ca}^{2+}-\mathrm{lo}-$ nen aktiviert $(32,33,34)$. Thrombin induziert jedoch überdies die Umwandlung von Fibrinogen zu Fibrin, welches einerseits mit dem synthetischen Substrat konkurrieren könnte, andererseits können die Fibrinpolymere zu einer Zunahme der Trübung führen, welche die Detektion des $\mathrm{NADH}-$ Verbrauchs bei $340 \mathrm{~nm}$ stört.

Dieser Störfaktor könnte durch Ausfällen des in der Probe vorhandenen Fibrinogens eliminiert werden. Der Ausfällungsschritt ist jedoch in zweifacher Hinsicht pro-

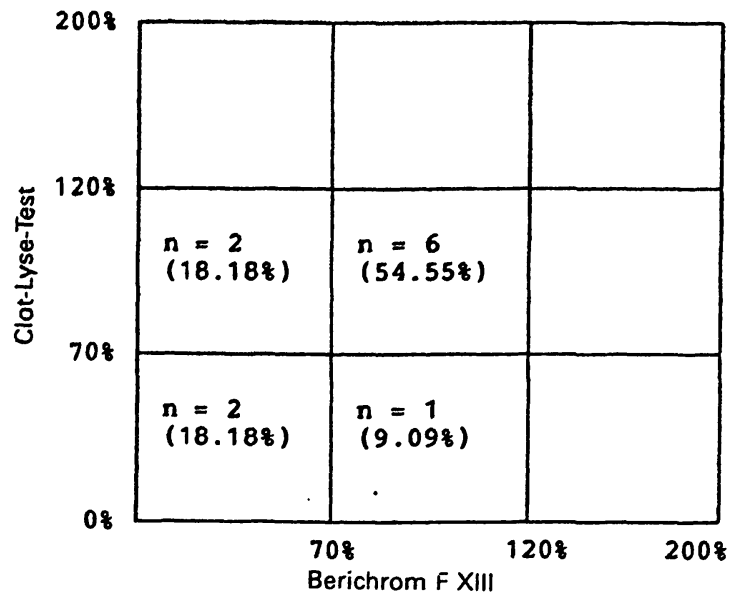

Abb. 7: Auswertung der mit Berichrom F XIII und mit dem Clot Lyse-Test bestimmten F XIII-Werte von 11 Plasmen mit erhöhtem $\mathrm{NH}_{3}$-Gehalt (>90 $\left.\mu \mathrm{g} / \mathrm{dl}\right)$ mit einer Neunfeldertafel. Die Grenzen $70 \%$ und $120 \%$ d. N. entsprechen dem Referenzbereich.

blematisch. Zum einen wird durch Fibrin die Aktivierungszeit von Faktor XIII durch Thrombin verkürzt 135 , 36), zum anderen wird dadurch häufig ein Teil des F XIII aufgrund seiner Affinität zu Fibrinogen mit ausgefällt. Letzteres wird auch durch Ergebnisse der vorliegenden Studie bestätigt. So findet man in den für den Clinisotest mit Bentonit defibrinierten Plasmen gegenüber unbehandelten Proben mit dem Berichrom-Test nur etwa $1 / 4$ der Faktor XIII-Aktivität. Die durch Fibrinogen verursachten Interferenzen wurden in dem von Fickenscher et al. (30) entwickelten Berichrom F XIII-Test durch einen neuen Inhibitor unterbunden, welcher die Quervernetzung des Fibrins und somit eine Zunahme der Trübung verhindert, jedoch nicht die Bildung von löslichem Fibrin. Fickenscher et al. (30) berichten, daß bis zu einem Fibrinogengehalt von $11 \mathrm{~g} / /$ kein unlösliches Fibrin nachweisbar war. Auch bei den von uns untersuchten Plasmen konnten keine durch den Fibrinogengehalt in der Probe verursachten Störungen gefunden werden.

Verfälschung der Meßergebnisse durch weitere mögliche Störfaktoren wie eine erhöhte Ammoniakkonzentration oder begleitende $\mathrm{NADH}$-abhängige. Reaktionen konnten bei unseren Bestimmungen nicht beobachtet werden.

Die Präzision sowohl in der Serie als auch von Tag zu Tag sowie die gute Linearität der Ergebnisse über den gesamten Meßbereich sprechen für die hohe Meßgenauigkeit der photometrischen F XIII-Bestimmung, so daß der Test als Einzelbestimmung durchgeführt werden kann.

Um einen schnellen Testablauf zu gewährleisten, wurde für den Berichrom F XIII-Test ein neues synthetisches Substrat ( $K 9$ ) entwickelt, welches eine hohe Umsatzrate und eine niedrige $K_{M}$ aufweist (30). Dies ermöglicht bei der manuellen Testdurchführung eine Faktor XIII-Bestimmung innerhalb von $15 \mathrm{~min}$, bei der automatisierten Version am Cobas Fara kann innerhalb von ca. 25 min die F XIII-Aktivität von 26 Proben bestimmt werden. Während die Testdurchführung beim Clot-Lyse-Test doch recht umständ́dich, langsam und personalintensiv ist, kann sie mit der Berichrommethode äußerst unkompliziert bei nur geringer Personalbindung durchgeführt werden.

Durch seine vielen Vorteile, die diese Arbeit zeigt, führt der Berichrom F XIII-Test zur einer enormen Erleichterung bei der Bewältigung der ständig steigenden Faktor XIIIAnforderungen. 
Schriftum:

1. Baer, U., Baukecht, J., Stangl, T., Háring, R. (1980) Verminderung von Wundheilungsstörungen durch prä- und postoperative quantitative Faktor XIII-Substitution. Zentralbl. Chir. 105, 642-651.

2. Benfer, J., Struck, H. (1979) Substitution von Faktor XIII bei Störungen der Wundund Knochenbruchheilung. Die gelben Hefte 19, 106.

3. Silberzahn, J. (1991) Faktor XIII and Wound-Healing Impairments in ENT. $2^{\text {nd }}$ internat. Conference on Factor XIII in Marburg.

4. Mishima, Y., Nagao, F., Ishibiki, K., Matsuda, M., Nakamura, N. (1984) Faktor XIII in der Behandlung postoperativer therapiefraktärer Wundheilungsstörungen. Chirurg 55 803-808.

5. Gierhake, F. W., Papastavrou, M., Zimmermann, K., Bohn, H., Schwick, H. G. (1974)

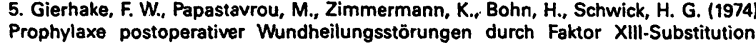
Dtsch. med. Wschr. 99, 1004-1009.

6. Pilger, E., Bertuch, H., Ulrich, A. \& Rainer, F. (1987) Capillary permeability in connective tissue disease: Influence of Fibrogammin P-therapy. Thromb. Haemostas. 58, 279

7. Guillevin, L., Chouvet, B., Mery, C., Godeau, P., Delbarre, F. (1982) Traitement de la sclérodermie généralisé par le facteur XIII. La Revue de Médicine interne, Tome III No 3, 273-277.

3. 273-277. . Lillevin, L. (1991) Prevention of Systemic Manifestations of Progressive Systemic 8. Guillevin, L. (1991) Prevention of Systemic Manifestations of Progressive Systemic
Sclerosis by Faktor XIII (Fibrogammine): Design of a Prospective Randomized Trial. $2^{\text {nd }}$ Internat. Conference on Faktor XIII in Marburg.

9. Henriksson, P., Hederr, U., Nilsson, I. M. (1977) Faktor XIII (Fibrin stabilizing factor) in Henoch-Schönlein's Purpura. Acta. Paediatr. Scan. 66, 273-277.

10. Kamitsuij, H., Tani, K., Yasui, M., Taniguchi, A., Taira, K., Tsukada, S., et al. (1987) Activity of blood coagulation factor XIII as prognostic indicator in patients with Schönlein-Henoch Purpura. Eur. J. Pediatr. 146, 519.

11. Kröniger, A., Seitz, R., Schwerk, W. B., Fickenscher, K., Arnold, R. \& Egbring, R. 11. Krōniger, A., Seik, R., Schwerk, W. B., Fickenscher, K., Arnold, R. Q Egbring, R. F XIII Substitution in Bleeding Complications. $2^{\text {nd }}$ Internat. Conference on Faktor XIII in Marburg.

12. Fukui, H., Kamitsuij, H., Nagao, T., Yamada, K., Akatsuda, J., Inagaki, M., et al. (1989) Clinical Evaluation of a Pasteurized Faktor XIII concentrate administration in $\mathrm{He}$ noch-Schönlein Purpura. Thromb. Res. 56, 667-675.

13. Suzuki, R., Toda, H. \& Takamura, Y. (1989) Dynamics of blood coagulation factor in ulcerative colitis and preliminary.study of the factor XIII concentrate. Blut 59, 162-164. 14. Galloway, M. J. Mackie, M. J. \& McVerry, B. A. (1983) Reduced levels of factor XIII in patients with chronic inflammatory bowel disease. Clin. lab. Haemat. 5, 427-428.

in patients with chronic inflammatory bowel disease. Clin. lab. Haemat. 5, 427-428.
15. Lorenz, R., Clemens, R., Karl, M., Classen, M. (1989) Substitution von F XIII-Konzen15. Lorenz, R., Clemens, R., Karl, M., Classen, M. (1989)
trat bei Colitis ulcerosa. Z. Gastroenterol. 2 (27), 89-90.

16. Wisen, O. \& Garlund, B. (1988) Hemostasis in Crohn's disease: Low Factor XIII levels in active disease. Scand. J. Gastroenterol. 23, 961-966.

17. Havemann, K., Egbring, R., Gropp, C., Schmidt, M., Sodomann, C. P. (1977) Faktor XIII-Mangel bei akuter Leukämie des Enwachsenen: Ergebnisse einer Substitution mit Faktor XIII. Klin. Wschr. $55801-809$.

18. Woitinas, F. (1991) Bleeding Disorder in Hypercellular Leukemia and Faktor XIII-Sub18. Woitinas, F. (1991) Bleeding Disorder in Hypercellular Leuken
stitution. $2^{\text {nd }}$ Internat. Conference on Factor XIII in Marburg.

stitution. ${ }^{\text {nd }}$ Internat. Conference on Factor XIII in Marburg.
19. Janzen, R. W. C., Bauer, W. \& Tilsner, V. (1982) The spontaneous course of factor XIII 19. Janzen, R. W. C., Bauer, W. \& Tilsner, V. (1982) The spontaneous course of factor XII
following intracranial hemorrhage: relevant for rebleeding? J. Neurosurg. Sci. 26, 135136.

20. Thie, A., Henze, Th., Degner, D., Oberling, M., Clemens, R., Klein, H. J., Lombard, G. F. \& Huchni, K. (1987) Factor XIII concentrate for prophylaxis of rebleeding in subarachnoidal hemorrage (SAH). Results of a prospective multicenter pilot study. Thromb. Haemostas. 58 (1), 513 .

21. Benfer, J. \& Struck, H. (1977) Factor XIII and fracture healing. Europ. Surg. Res. 9 21. Benfer,

22. Kugelmeier, K. (1985) Experimentelle proliferationskinetische Untersuchungen über die Wirkung des Plasmafaktor XIII auf das Knochenwachstum in vitro und in vivo. Diss. Ulm.

23. Claes, L., Burri, C., Gerngross, H., Mutschler, W. (1984) Die Beschreibung der Frakturheilung durch F XIIL Helv. Acta51, 201-212.
24. Gerngross, H., Steinmann, R., Graf, N. (1987) Faktor XIII und Knochenheilung, Med. Welt 38, 1533-1538.

25. Gerngross, H. \& Claes, L. (1991) Experimental Results in Factor XIII and Bone-Graft Healing. $2^{\text {nd }}$ Internat. Conference on Factor XIII in Marburg.

26. Dempfle, C. E., Hochreuther, K., Helmchen, G. \& Heene, D. L. (1991) Microtiter As say for Factor XIII Activity. $2^{\text {nd }}$ Internat. Conference on Factor XIII in Marburg.

27. Selmayr, E., KIöss, H. R. \& Müller-Berhaus, G. (1991) A New Method for the Determination of Factor XIII Activity Using Bionylated Fibrinogen. $2^{\text {nd }}$ Internat. Conference on Factor XIII in Marburg.

28. Lee, K. N., Birkbichler, P. S. \& Patterson jr., M. K. (1988) Colorimetric assay of blood coagulation factor XIII in plasma. Clin. Chem. 34, 906-910.

29. Muszbek, L., Polgár, J. \& Fésüs, L. (1985) Kinetic Determination of Blood Coagula tion Factor XIII in Plasma. Clin. Chem. 31,35-40.

30. Fickenscher, K., Aab, A. \& Stüber, W. (1991) A Photometric Assay for Blood Coagulation Factor XIII. Thromb. Haemostas. 65 (5), 535-540.

31. Karges, H. E., Heimburger, N. (1978) Gerinnungsfaktor XIII: Schnelltest bei Defizitverdacht. Laboratoriumsblätter $28,71-78$.

32. Takagi, T. \& Doolittle, R. F. (1974) Amino acid sequence studies on factor XIII and the peptide released during its activation by thrombin. Biochemistry 13,750 .

33. Henriksson, P. \& McDonagh, J. (1983) Factor XIII activation and interactions. In: Factor XIII and Fibronectin. (R. Egbring, H. G. Klingemann eds.) Medizinische Verlagsgesellschaft (Marburg) pp 1-14.

34. Curtis, C. G., Brown, K. L., Credo, R. B., et al. (1974) Calcium dependent unmasking of active center cysteine during activation of fibrin stabilizing factor. Biochemistry 13

35. Credo, R. B., Curtis, C. G. \& Lorand, L. (1978) $\mathrm{Ca}^{2-}$ related regulatory function of fibrinogen. Proc. Natl. Acad. Sci. USA 75, 4234.

36. Janus, T. J., Lewis, S. D., Lorand, L. \& Shafer, J. A. (1983) Promotion of thrombin catalyzed activation of factor XIII by fibrinogen. Biochemistry 22, 6269-6272.

Anschrift der Verfasser:

Dr. Eva Solleder

Dagmar Demuth

Christian Pfeiffer

Dr. Michael Bomhard

Dr. Johannes Mayer

Dipl.-Chem. Thomas Eller

Dr. Peter Brauer

Prof. Dr. Franz Keller

Zentrallabor der Medizinischen Universitätsklinik

Josef-Schneider-Straße 2

8700 Würzburg

Dr. Joachim Grün

Dr. Karl Fickenscher

Dr.'Carola Wagner

Behringwerke AG

Postfach 1140

3550 Marburg 